\title{
Testing Smart City environmental monitoring technology using small scale temporary cities
}

\author{
Steven J. Johnston*†, Philip J. Basford*, Florentin M. J. Bulot*, Natasha H. C. Easton ${ }^{\ddagger}$, Gavin L. Foster ${ }^{\ddagger}$, \\ Matthew Loxham ${ }^{\S}$, Mihaela Apetroiae-Cristea*, Andrew K. R. Morris ${ }^{\top}$, Simon J. Cox*. \\ * Faculty of Engineering and the Physical Sciences, University of Southampton, UK \\ $\ddagger$ Faculty of Environmental and Life Sciences, University of Southampton, UK \\ $\S$ Faculty of Medicine, University of Southampton, UK \\ I National Oceanography Centre, Southampton, UK \\ †sjj698@zepler.org
}

\begin{abstract}
Exposure to Particulate Matter (PM) has been identified as a major health problem worldwide. Established measurement techniques require equipment costing many thousands of dollars and specialist expertise to maintain. Ongoing research is investigating the use of low cost $<\$ 300$ sensors to enable greater temporal-spatial density of readings to be taken. There are questions about the suitability and reliability of these lowcost sensors, which can be addressed by deploying and evaluating the sensors in real world applications. Rather than deploying standalone data loggers for each sensor, each air quality monitor is connected to an IoT device to enable real time transmission of data. We propose festival sites as small scale cities to enable a short term deployments and evaluation of sensors. This work illustrates that, if coupled with higher resolution of wind data, low-cost sensors may enable to follow the evolution of pollution hotspots and help the identification of pollution sources. This study, building upon the body of work focused on the evaluation and best practice of using low-cost sensors for PM monitoring. We present data from these IoT devices and experiences gained from using a festival site as a substitute for a city.
\end{abstract}

Keywords-Air quality, Internet of Things, Environmental monitoring

\section{INTRODUCTION}

Exposure to poor air quality is a concern for public health worldwide associated with between 4.2 million to 8.9 million deaths per year worldwide in 2015 [1], [2], [3], [4]. Exposure to air pollution is also linked to a range of adverse health conditions. One contributor to air pollution is Particulate Matter (PM). Particles of dust with an aerodynamic diameter $<2.5 \mu \mathrm{m}$, known as $\mathrm{PM}_{2.5}$, are estimated to be associated with 29,000 deaths per year in the UK [5]. PM is usually monitored by a network of reference monitoring stations. These stations are costly, require significant expertise to be operated and finding locations for these stations can be challenging in built-up areas, leading to a low density of deployment with some cities not having any monitoring stations. Low-cost PM sensors combined with Internet of Things (IoT) technologies may provide a solution to these limitations [6]. The research on these low-cost PM sensors is still at an early stage and this paper presents the experiences gained when testing such sensors as part of an IoT deployment at a festival.

978-1-5386-4980-0/19\$31.00 (c) 2019 IEEE
In this paper, a festival site is considered as a small temporary city and is used to prototype a new IoT system, with some of the challenges posed by a full scale deployment. It enables evaluation of the complete devices, sensors and IoT infrastructure. It engages the population with the research being conducted and simulates a city-scale deployment including continuous and intermittent measurements. The objectives of this study are: (1) to create a network of low-cost PM monitors analogous to a city deployment; (2) the incorporation of IoT technologies in the network to enable scalability and construction by non-specialists; (3) to conduct a case study of PM network made from low-cost sensors to determine their capacity to monitor and track transient and local PM events. Together these demonstrate a scalable low cost PM sensor network that augments existing city sampling, delivering high spatial and temporal resolution that can lead to the identification of particular PM events and their origin - for a better safeguarding of human health in cities.

A brief overview of air quality monitoring is presented in Section II. A description of the festival used to present the work at is given in Section III. Details of the equipment deployed for both the workshops and continuous logging of air quality on the site are presented in Section IV, with results presented in Section V and discussed in Section VI. Finally we present our conclusions in Section VII.

\section{AIR QUALITY MONITORING}

Air quality varies spatially [7] and the concentrations observed at a reference monitoring station may not be representative of actual exposure to air pollution. To assess personal exposure and to identify pollution sources different approaches need to be combined [8]. Low-cost sensors are suitable to be deployed in a large number providing spatially dense information about air quality. The quality of the data collected by these sensors is still under investigation.

Over the last year, a number of community or commercially lead projects around air quality have emerged [9] in different countries engaging citizens with air quality monitoring. These projects rely on low-cost sensors and facilitate their deployment in large numbers. The data from these sensors needs to be collated and processed, this can be achieved 


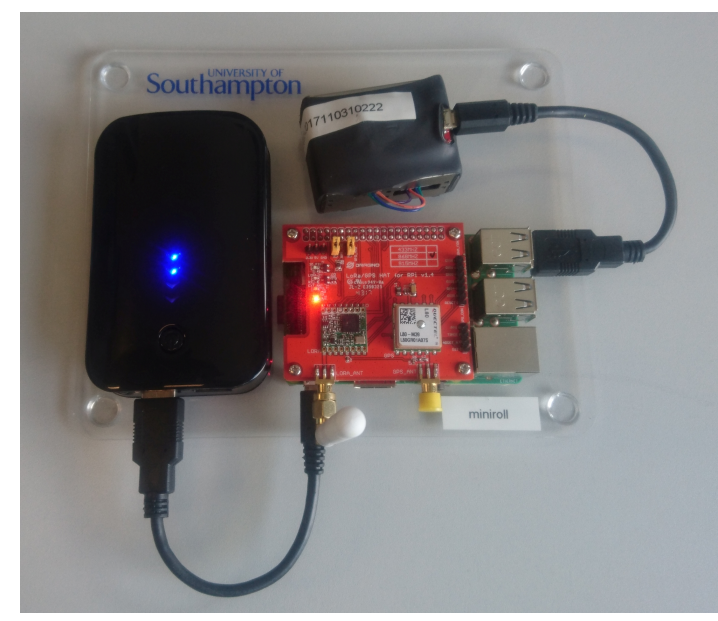

Fig. 1. One of the air quality monitors used for the workshops, and subsequently deployed around the festival site.

by combining the air quality sensors with IoT technologies such as LoRaWAN to provide data connectivity. It embeds scalability to a created network and when designed with open access elements traditional prohibitive barriers to participation are absent. These properties of IoT technology were included in this work to deliver a study that is representative of a PM network that could be operated by citizen scientists and built upon over time.

\section{Electromagnetic FiELD 2018}

Electromagnetic Field (EMF) (https://www.emfcamp.org/) is a bi-annual camping festival, run by volunteers on a notfor-profit basis. In 2018 over 2000 people attended the festival which took place in Eastnor Castle Deer Park, UK, between $31^{\text {st }}$ August and $2^{\text {nd }}$ September. The event was held on a greenfield site, with very limited permanent infrastructure. A week before the event a team of volunteers arrived and installed the infrastructure that would be found in an urban environment including: power distribution and network infrastructure. Each camping pitch is provided with mains electricity and gigabit Ethernet. Also installed on the site were sanitation facilities, WiFi and GSM networks.

The site opened to attendees on Thursday afternoon and the festival started at 13:00 on Friday, the festival finished at midnight on Sunday, with attendees vacating by 12:00 Monday. During the festival the site was a largely vehicle free area (only all-terrain vehicles used by volunteers were allowed). The small physical size and easy access to all areas reduced deployment and maintenance complexities. The festival is different to a permanent city in respect of its light traffic, no building and different sources of pollution. It is a good test of setting up a PM monitoring sensor network, demonstrating IoT infrastructure and data collection from nonspecialists.

\section{MATERIALS AND METHODS}

The festival provided an opportunity to run workshops, teaching participants to use and program low-cost PM sen-
TABLE I

KEY COMPONENTS OF THE AIR QUALITY MONITORS. PRICES CORRECT NOVEMBER 2018

\begin{tabular}{cc} 
Item & Price (USD) \\
\hline Raspberry Pi & 35 \\
SD card & 20 \\
Plantower PM Sensor & 20 \\
FTDI USB-serial & 16 \\
Dragino LoRaWAN HAT & 36 \\
PiFace RTC & 10 \\
RTC Battery & 1 \\
USB Power bank & 25 \\
USB cables (2) & 2 \\
\hline Total & 165
\end{tabular}

TABLE II

LORAWAN GATEWAY USAGE STATISTICS. FOR LOCATION SEE FIGURE 3. GATEWAY EMF3 WAS ONLY DEPLOYED DURING THE WORKSHOPS.

\begin{tabular}{crr} 
Gateway & Messages Received & Messages Transmitted \\
\hline EMF1 & 17,846 & 44 \\
EMF2 & 17,796 & 23 \\
EMF3 & 910 & 0
\end{tabular}

sors, that are able to transmit the readings gathered over a LoRaWAN network. Having run the workshops on the first day of the festival, we deployed the sensors around the site to complement the purpose built sensors deployed beforehand.

\section{A. Hardware}

The hardware used was designed to be easily re-producible, to enable workshop attendees to replicate it at home. The main components included in the device are listed in Table I, and are mounted on a laser cut acrylic sheet as shown in Figure 1. Instead of using an arbitrary numerical string to identify each device; the devices were named after confectionery.

A Raspberry Pi was chosen for the main processor of the device because of its popularity in the hobbyist community and our positive experiences using it for previous air quality deployments [6]. The Plantower PM sensors have also shown to be reliable in the same deployment.

The device is battery powered as the availability of power distribution in the workshop area could not be guaranteed, it also meant that when the devices were deployed around the site more flexibility was available to choose locations.

Three LoRaWAN gateways forwarding data to the The Things Network (https://www.thethingsnetwork.org/) were deployed and openly accessible. Other $3^{\text {rd }}$ party gateways were also deployed on site providing additional redundancy. This also means that there were other people using the LoRaWAN network, which is reflected in the amount of traffic observed through the gateways as shown in Table II. Gateway EMF3 was only deployed for the duration of the workshops in the corner of the venue. Gateway EMF1 was deployed at the top of a mast towards the North edge of the site, at an estimated height of $6 \mathrm{~m}$ above ground level see Figure 2(b). Gateway EMF2 was installed on a mast towards the South-East of the camp site, at an approximate height of $8 \mathrm{~m}$ above ground level. See Figure 3 for details of gateway locations. 

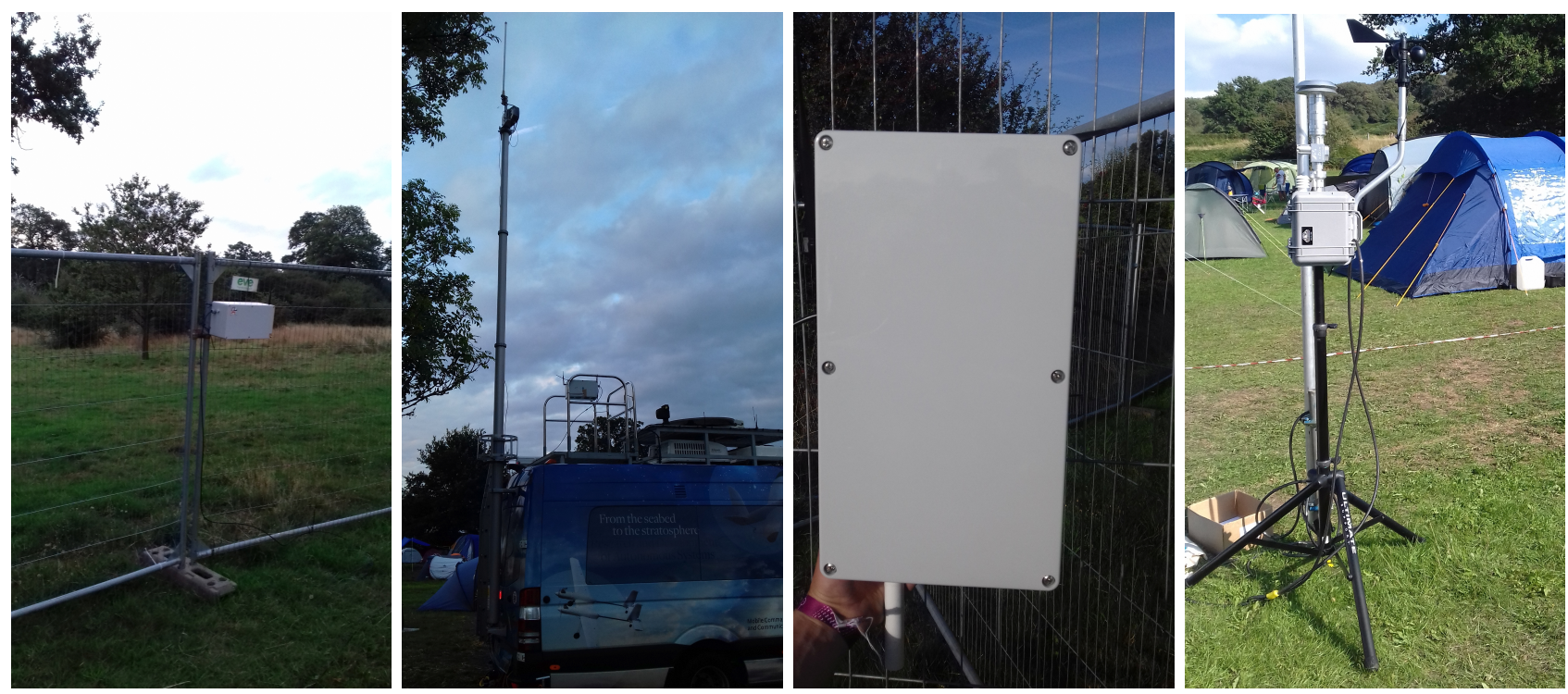

Fig. 2. Pictures of the deployment locations around the site, see Figure 3 for locations.

(a) Air quality monitor B1-EMF, (b) Air quality monitor B2-EMF and LoRaWAN gateway EMF1, (c) Air quality monitor B3-EMF (d) ARA N-FRM Sampler.

\section{B. Software}

Python 3 was used for interacting with the sensors and LoRaWAN network and all the user interaction was through a Jupyter notebook instance which is commonly used for teaching [10]. The custom PM sensor library is available in pypi (https://pypi.org/project/plantower/). To make it as easy as possible for people to recreate the workshops at home the standard Raspbian Operating System (OS) was used. With Raspbian there is a risk of SD card corruption if the power is lost during a file system write [11], an occurrence that is more likely when running on battery power.

During the workshops data was visualised using Cayenne (https://cayenne.mydevices.com), and efficiently encoded using the Cayenne-LPP format [12].

\section{Workshop}

The attendees worked through the exercise in lock-step with the presenter. The workshops started with a brief summary of why air quality is an important issue, why current monitoring is inadequate and how the low cost PM sensors can address these problems. The workshops then explained how to read the PM sensor, how to transmit LoRaWAN messages, and how to visualise the data. The workshops were led by a presenter and two other experts were available to help the audience. There were a wide variety of different abilities present and attendees were encouraged to extend exercises once completed.

\section{Deployment}

Three air quality monitors, named B1-EMF, B2-EMF and B3-EMF were deployed at the festival the morning before the workshops (31 ${ }^{\text {st }}$ August). B3-EMF contained a Honeywell HPMA115S0, a Plantower PMS5003, a Plantower PMS7003 and an Alphasense OPC-N2 as described in a previous study
[6]. B1-EMF and B2-EMF are an upgraded version of B3EMF and can host up to ten sensors [13]. For this deployment, only a single Plantower PMSA003 was added. An ARA NFRM Sampler from ARA Instruments was deployed $\approx 10 \mathrm{~m}$ away from B2-EMF at a height of $1.8 \mathrm{~m}$ above ground level. This sampler collects PM on a filter and was equipped with a size selective inlet to collect only $\mathrm{PM}_{2.5}$. The filter was preweighed and was weighed again after the festival to obtain the average concentration of $\mathrm{PM}_{2.5}$. The $\mathrm{N}-\mathrm{FRM}$ samples $1 \mathrm{~m}^{3} / \mathrm{h}$, and enables PM to be directly weighed rather than inferred as performed in the low-cost sensors. The sampler is powered by rechargeable batteries and also contains a modified lowcost PM sensor, Shinyei PPD42NS, temperature and humidity sensor, and wind speed and direction sensor.

After the workshops, 13 nodes were deployed around the site to increase the spatial resolution of the PM data gathered. Two different Plantower sensors were used, 11 of the nodes were equipped with a Plantower PMS5003 and two were equipped with a Plantower PMSA003. The workshop were used to test the sensors prior to the deployment and to identify faulty sensors. Ten of the nodes were battery powered and three were mains powered. The locations of the nodes are shown on Figure 3. The inclusion of the Real Time Clock (RTC) in the nodes, guaranteed reliable timestamped data could be collected without requiring network connectivity. These systems recorded all data locally and transmitted hourly averages using LoRaWAN which were then made available to all attendees of the event. The power demands of the sensor and Raspberry Pi meant that uninterrupted data collection was not possible with the battery packs available.

The sampler simulated a reference instrument, the three air quality monitors simulated intermediate quality instruments and the individual nodes simulated low quality instruments; replicating a typical smart city scenario. 


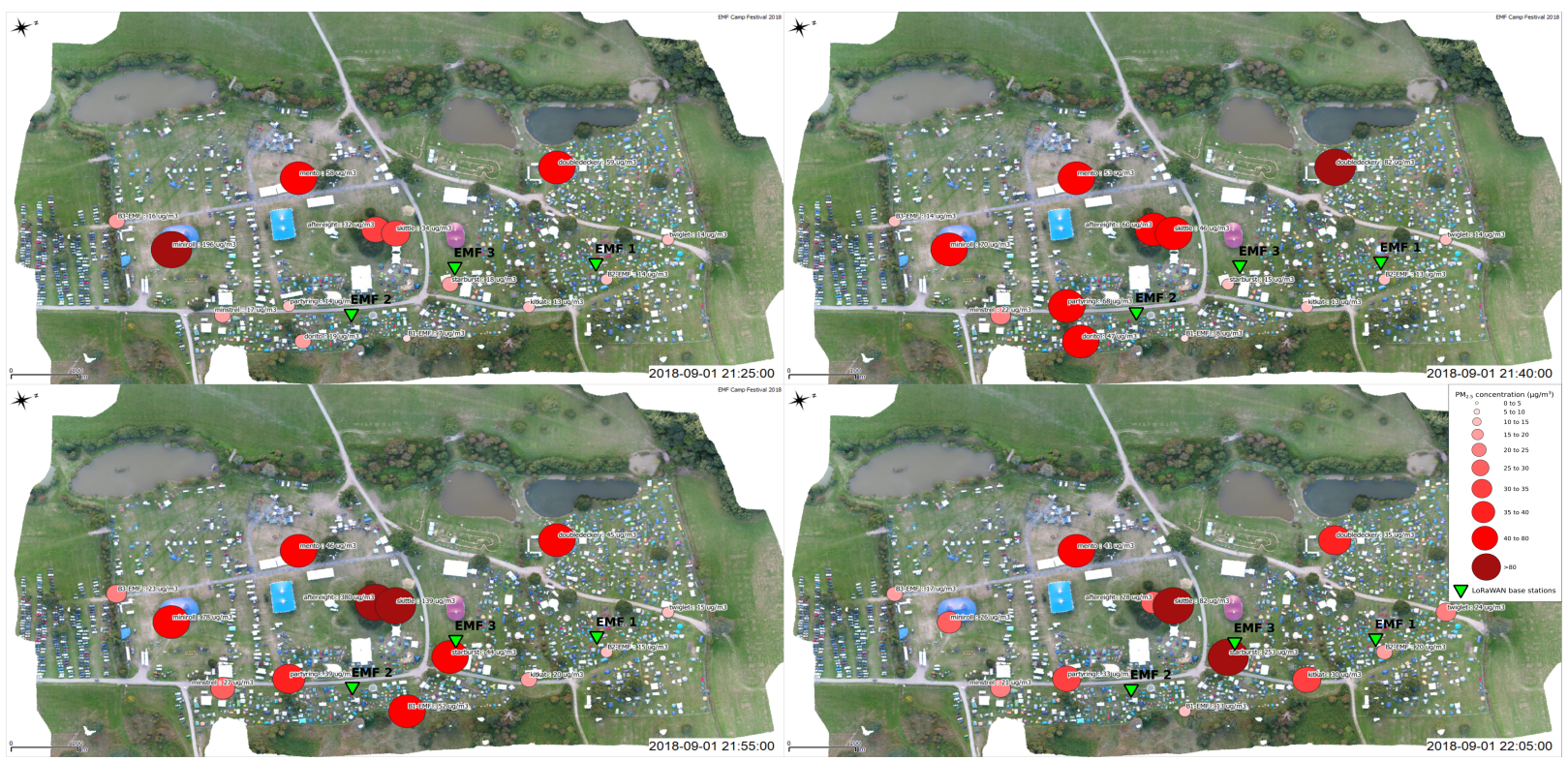

Fig. 3. Snapshots of the time-lapse video of the measurements recorded by all the sensors during the festival. Sensors shown at their deployment location. For the air quality monitors, the mean value of all the sensors hosted has been used. The full time-lapse is available in the supporting dataset.

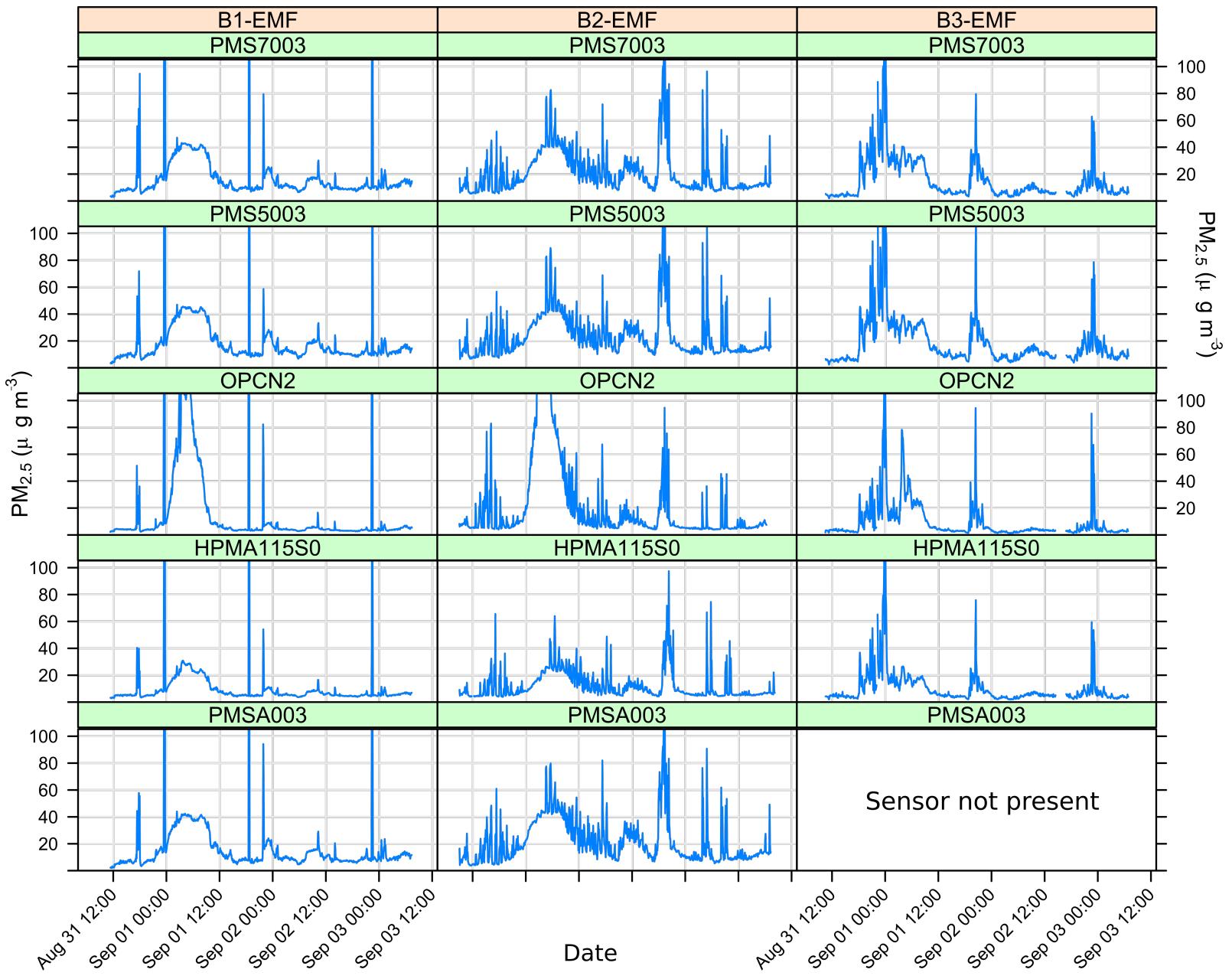

Fig. 4. Time series charts of the Honeywell HPMA115S0, Alphasense OPC-N2, Plantower PMS5003, Plantower PMS7003 and Plantower PMSA003 in the three Air Quality Monitors deployed during the festival, see Figure 3 for locations. 


\section{REsults}

The sampler was operational for a total of $67 \mathrm{~h} 40 \mathrm{~min}$ during which it collected $600.2 \mu \mathrm{g}$ of $\mathrm{PM}_{2.5}$ which corresponds to an average of $8.85 \mu \mathrm{g} / \mathrm{m}^{3}$ during the festival. The modified Shinyei PPD42NS reported a mean concentration of $5.1 \mu \mathrm{g} / \mathrm{m}^{3}$ showing that the sensor underestimated the concentration of $\mathrm{PM}_{2.5}$. The mean concentrations reported by B2-EMF sensors located $\approx 10 \mathrm{~m}$ away from the sampler and $\approx 2.5 \mathrm{~m}$ above the ground ranged from $12 \mu \mathrm{g} / \mathrm{m}^{3}$ to $24 \mu \mathrm{g} / \mathrm{m}^{3}$.

Figure 4 presents the time series of the sensors installed in the three air quality monitors deployed at the festival. Each model of sensor shows similar variations in each air quality monitor confirming the results of previous studies on the same sensors [6], [13]. The three Plantower models report similar values within each box with average $\mathrm{PM}_{2.5}$ concentrations reported ranging from $17 \mu \mathrm{g} / \mathrm{m}^{3}$ to $19 \mu \mathrm{g} / \mathrm{m}^{3}$ for $\mathrm{B} 1-\mathrm{EMF}$, from $21 \mu \mathrm{g} / \mathrm{m}^{3}$ to $24 \mu \mathrm{g} / \mathrm{m}^{3}$ for B2-EMF and from $15 \mu \mathrm{g} / \mathrm{m}^{3}$ to $18 \mu \mathrm{g} / \mathrm{m}^{3}$ for B3-EMF . The Honeywell HPMA115S0 and the Alphasense OPC-N2 report lower values than the three Plantower (with average $\mathrm{PM}_{2.5}$ concentrations reported ranging from $10 \mu \mathrm{g} / \mathrm{m}^{3}$ to $12 \mu \mathrm{g} / \mathrm{m}^{3}$ and from $10 \mu \mathrm{g} / \mathrm{m}^{3}$ to $21 \mu \mathrm{g} / \mathrm{m}^{3}$ respectively). The Alphasense OPC-N2 shows higher response to peaks of $\mathrm{PM}_{2.5}$ than the four other sensors. On $1^{\text {st }}$ September around 00:00 a peak of pollution appeared on B3-EMF, a peak of pollution then appears on B1-EMF around 03:00 and shortly after on B2-EMF. During this period of time, the wind sensor of the sampler reported an average wind speed of $0.07 \mathrm{~m} / \mathrm{s}$ and a predominant Northerly wind direction, suggesting a single pollution event.

Figure 5 presents the Box-Whisker plot for each of the 12 individual sensors deployed around the festival. One of the 13 nodes deployed did not record any data during the festival. The sensors shows a different spread of concentration compared to each other. The low variability observed with the Plantowers deployed in the three air quality monitors suggests that the variability observed among the 12 sensors is linked to different pollution exposure from nearby pollution sources. The lowest values observed are for Partyring and Minstrel located in a quieter area, away from the smoke machines at the festival, and $60 \mathrm{~m}$ from each other. Lower values than average were also observed for Kitkat located in a crèche marquee. The node reporting the highest values was Mento, located $50 \mathrm{~m}$ away from the main stage of the festival which was equipped with flame throwers and smoke machines. Jaffacake, located $100 \mathrm{~m}$ from the main stage, also reported higher values than the rest of the nodes. Figure 3 shows snapshots of the time-lapse of the measurements taken at each location during the festival between 21:25 and 22:05 on the $1^{\text {st }}$ September. A video of the time-lapse for the full duration of the festival is available in the underlying dataset. These snapshots show the dispersion of pollution hotspots and potentially enable identification of pollution sources, if coupled with higher resolution wind data.

\section{DISCUSSION}

The accuracy of low-cost PM sensors is affected by the composition and the size of PM [14], [15], they correlate

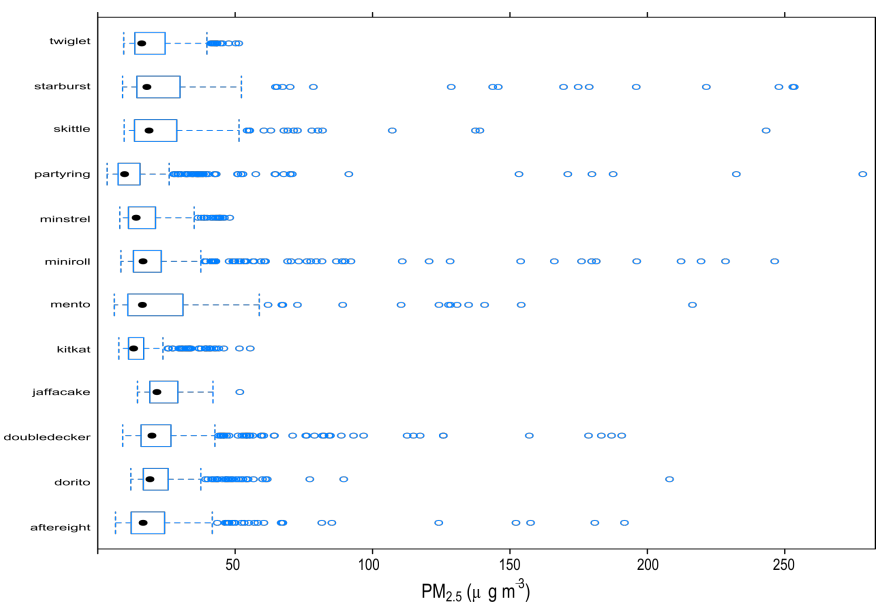

Fig. 5. Box-Whisker plot for the 12 individual sensors deployed around the festival site that provided data, see Figure 3 for locations. Only the lower $99 \%$ percentile data is shown.

better with reference instruments for higher levels of pollution (above $10 \mu \mathrm{g} / \mathrm{m}^{3}$ to $20 \mu \mathrm{g} / \mathrm{m}^{3}$ ) [16], [17]. They report different values depending on environmental factors such as relative humidity and to a lesser extent temperature which may be corrected [17], [18], [19]. While these sensors are suitable for tracking pollution variations, the actual values they report are questionable and on-site calibration is required before deploying them to address their variability towards PM composition and size [14]. These steps require expertise and increase the cost of the deployment. We recommend that the sensors are at least collocated together in a confined area for at least a few hours to apply a correction coefficient based on the mean value obtained for all the sensors.

The scope of this study was to test the viability of monitoring air quality in a small scale temporary city and the above mentioned calibration was not conducted. The sensor results obtained are suggestive and a more robust study is required. This study enabled a comparison between sensor characteristics, such as their sensitivity to peak concentrations, their tendency to over report and intra-model variability. The results obtained suggest that by combining the data obtained by low-cost PM sensors with meteorological data, it may be possible to track events of pollution and to infer the location of emission sources; this is not currently possible through the centralised PM observations within cities. The case study demonstrates one of the ways low-cost sensors can deliver improved PM monitoring and better safeguard human health. Future work is needed to better correct environmental factors to get more accurate PM concentrations from low-cost sensors.

The Raspberry Pi power issues illustrate intermittent data capture that could be experienced during city-wide monitoring studies. Out of the twenty sensors used during the workshops at least one reported abnormally high readings compared to the other sensors, revealing the possibility of faulty sensors and the need to compare the sensors between each other before deployment. The sensor is suspected to have been damaged during assembly. 
Using a festival site as a proxy for a city enabled the devices to be deployed in a realistic environment and to gather valuable data. This reduced the size of the site when compared to a real city improving access to each of the sensors deployed; an ideal environment for testing early stage hardware / software. LoRaWAN was used for the transmission of data from the nodes, although this could also have been achieved using the site-wide WiFi network to which the nodes were also connected. This WiFi link would not be available in final deployment scenarios but it enabled bug fixes and updates to be deployed. The non-waterproof nature of some nodes was a concern, addressed by the careful choice of sheltered deployment locations. The temporary city provided an ideal location to test these new air quality sensors and highlighted issues that could be fixed more easily than if found in a citywide deployment scenario.

\section{CONCLUSION}

The low variability observed between the sensors deployed in the air quality monitors shows their potential to provide useful information regarding air quality in a city. Future work is needed to better correct environmental factors to get more accurate PM concentrations from low-cost sensors. We recommend, as a minimum calibration procedure, that the sensors are collocated together in a confined area for at least a few hours to apply a correction coefficient based on the mean value obtained for all the sensors. Festival activities, which generated different pollution and spreads of concentration are registered by the individual sensors suggesting the possibility of monitoring the impact of varied activities on air pollution in a city. The time-lapse presented in the underlying dataset reveals that it is possible to display the data generated in a meaningful manner for example for citizen science applications. This time-lapse also illustrated the sensor's capacity to track events of pollution and to infer the location of emission sources. The LoRaWAN transmission could be used in future deployments to produce this time-lapse in real time. This IoT deployment in a festival had many similarities with a city-scale deployment and enables validation of environmental monitoring equipment, while at the same time being easier logistically than a full scale test deployment. The diversity of the activities at the festival replicated the variety of activities of a city. We conclude that small scale temporary cities are a good test bed for environmental monitoring studies.

The underlying datasets and workshop instructions are available from: https://doi.org/10.5281/zenodo.2578171.

\section{ACKNOWLEDGEMENTS}

We acknowledge funding from the following sources: Engineering and Physical Sciences Research Council; Next Generation Unmanned Systems Science Centre for Doctoral Training supported by the Natural Environmental Research Council [NE/L002531/1]; the Leverhulme Trust through the Southampton Marine and Maritime Institute; Engineering and Physical Sciences Research Council [EP/P004024/1]; University of Southampton Public Engagement with Research unit (PERu).
We thank: Charlie Davenport for designing and building workshop devices; SotonUAV, \& AMSAT-UK for their support in locating the LoRaWAN gateways; Tanya Ossont for her support with logistics; the EMF organising committee.

\section{REFERENCES}

[1] P. J. Landrigan, R. Fuller, N. J. Acosta et al., "The Lancet Commission on pollution and health," October 2017.

[2] A. J. Cohen, M. Brauer, R. Burnett et al., "Estimates and 25-year trends of the global burden of disease attributable to ambient air pollution: an analysis of data from the Global Burden of Diseases Study 2015," The Lancet, vol. 389, no. 10082, pp. 1907-1918, may 2017.

[3] M. Brauer, G. Freedman, J. Frostad, and A. e. a. van Donkelaar, "Ambient Air Pollution Exposure Estimation for the Global Burden of Disease 2013," Environmental Science \& Technology, vol. 50, no. 1, pp. 79-88, jan 2016.

[4] R. Burnett, H. Chen, N. Fann et al., "Global estimates of mortality associated with long- term exposure to outdoor fine particulate matter," PNAS, vol. 115, no. 38, 2018.

[5] The Royal College of Physician, "Every breath we take: the lifelong impact of air pollution. Report of a working party," Royal College of Physicians, Tech. Rep. February, 2016.

[6] S. J. Johnston, P. J. Basford, F. M. J. Bulot et al., "IoT deployment for city scale air quality monitoring with Low Power Wide Area Networks," in Global IoT Summit, May 2018.

[7] S. L. Greco, A. M. Wilson, J. D. Spengler, and J. I. Levy, "Spatial patterns of mobile source particulate matter emissions-to-exposure relationships across the United States," Atmospheric Environment, vol. 41, no. 5, pp. 1011-1025, 2007.

[8] J. J. West, A. Cohen, F. Dentener et al., "What we breathe impacts our health: Improving understanding of the link between air pollution and health," Environmental Science \& Technology, vol. 50, no. 10, pp. 4895-4904, May 2016.

[9] L. Morawska, P. Thai, X. Liu et al., "Applications of low-cost sensing technologies for air quality monitoring and exposure assessment: how far have they gone?" Environment International, vol. 116, pp. 286-299, July 2018.

[10] A. A. Smith, "Teaching computer science to biologists and chemists, using jupyter notebooks: tutorial presentation," Journal of Computing Sciences in Colleges, vol. 32, no. 1, pp. 126-128, 2016.

[11] P. Basford, G. Bragg, J. Hare et al., "Erica the Rhino: A Case Study in Using Raspberry Pi Single Board Computers for Interactive Art," Electronics, vol. 5, no. 3, p. 35, June 2016.

[12] “Cayenne LPP 2.0.” [Online]. Available: https://community.mydevices. com/t/cayenne-lpp-2-0/7510

[13] S. Johnston, P. J. Basford, F. Bulot et al., "City scale particulate matter monitoring using LoRaWAN based air quality IoT devices," Sensors, January 2019.

[14] A. C. Rai, P. Kumar, F. Pilla et al., "End-user perspective of lowcost sensors for outdoor air pollution monitoring," Science of the Total Environment, vol. 607-608, pp. 691-705, December 2017.

[15] S. Sousan, K. Koehler, L. Hallett, and T. M. Peters, "Evaluation of the Alphasense optical particle counter (OPC-N2) and the Grimm portable aerosol spectrometer (PAS-1.108)," Aerosol Science and Technology, vol. 50, no. 12, pp. 1352-1365, 2016.

[16] K. E. Kelly, J. Whitaker, A. Petty et al., "Ambient and laboratory evaluation of a low-cost particulate matter sensor," Environmental Pollution, vol. 221, pp. 491-500, February 2017.

[17] T. Zheng, M. H. Bergin, K. K. Johnson et al., "Field evaluation of low-cost particulate matter sensors in high- and low-concentration environments," Atmospheric Measurement Techniques, vol. 11, no. 8, pp. 4823-4846, 2018.

[18] R. Jayaratne, X. Liu, P. Thai et al., "The influence of humidity on the performance of a low-cost air particle mass sensor and the effect of atmospheric fog," Atmospheric Measurement Techniques, vol. 11, no. 8, pp. 4883-4890, 2018.

[19] L. R. Crilley, M. Shaw, R. Pound et al., "Evaluation of a low-cost optical particle counter (Alphasense OPC-N2) for ambient air monitoring," Atmospheric Measurement Techniques, vol. 11, no. 2, pp. 709-720, 2018. 